\title{
Location and Cartographic Integration for Multi-Providers Location Based Services
}

\author{
Roula Karam, Frank Favetta, Rima Kilany, Robert Laurini \\ LIRIS CNRS UMR 5205, INSA de Lyon, Université de Lyon, F-69621 Villeurbanne, France \\ ESIB, Faculté d'Ingénierie, Universite Saint Joseph, B.P:11-514, Beirut, Lebanon \\ Naval Academy Research Institute, Lanveoc-Poulmic, BP 600, 29240, Brest Naval, France \\ \{Roula.karam@insa-lyon.fr; Roula.karam@ecole-navale.fr; Franck.favetta@insa-lyon.fr; \\ Rima.kilany@usj.edu.lb; Robert.laurini@insa-lyon.fr\}
}

\begin{abstract}
Since the development of Geographic Information Systems (GIS) and the increasing popularity of mobile devices, many standardization bodies such as OGC (Open Geospatial Consortium) had proposed the implementation of Location Based Services (LBS) applications. Furthermore, map service providers started to use the correspondent devices for visualizing geographic data essentially based on the users' location, his contextual information and his profile. However, many geographic databases (GDBs) could offer slightly different data/metadata for the same requested service. Advanced LBS have to improve interoperability among them. In this paper, we had elaborated many reasoning algorithms and build/match extended geo-ontology framework for the integration of homologous objects on mobile devices. Our approach is illustrated by a tourism LBS case study and could be applied for any geographic domain where position, place name, semantic details and visual aspects' (icon, texture, color, etc.) ambiguities will be integrated automatically through our platform.
\end{abstract}

\section{Introduction}

Nowadays, the co-existence of many and heterogeneous geographic databases covering the same area, implies to study how these data/metadata should be integrated in order to avoid duplicated results on the screen. Interoperability is the key aspect to elaborate in such situation. It is defined by OGC as the "capability to communicate, execute programs, or transfer data among various functional units in a manner that requires the user to have little or no knowledge of the unique characteristics of those units" (OpenGIS, 1996).

At the application level, a motivating example, as shown in the figurel below, had inspired us to direct our study towards a major problem which is related to location and cartographic integration of same geo-located service from many provid- 
ers. Let us consider a user's request to find the nearest restaurant in his area. First of all, he might encounter the answer of an American restaurant listed by two different providers, not exactly located at the same place (50 meters of difference). The same restaurant is named "the Roadster Diner" in the first one and "Roadster" in the second one, with few differences in their semantic details and represented differently as cartographic symbols (icons) on different proprietary base maps. This inconsistency in spatial and non-spatial information from both providers is a fact and we must know how to deal with it in order to visualize both as the same integrated object.

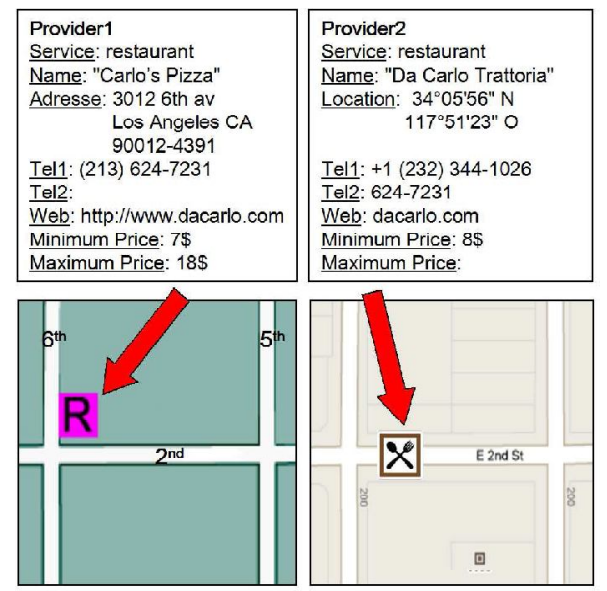

Fig. 1 Example of the same LBS restaurant from two providers (candidates for integration)

In order to solve most of the problems related to the use case scenario listed above, we propose a fully interoperable system with the following components:

- $\quad$ Subsystem for Location Integration: using specified algorithms for geographic and place names' integration such as Euclidian and Levenshtein distances and a semantic ontology reasoner via Protégé for their semantic details integration.

- Subsystem for Cartographic Integration: using a new type of geoontology, named CartOntology, for formalizing explicit knowledge about our domain of interest (touristic points of interests POI) and adjusting the map conflation, semantically and visually, based on the user profile's ontology, his geographic zone or context information and the graphical semiology constraints.

This paper is structured as follows: Section 2 provides a description of the problems being raised from the use case scenario. Section 3 presents an overview of the related works. In Section 4, we discuss and evaluate our contribution. Sec- 
tion 5 details all the implemented solutions for location and cartographic integrations. Section 6 concludes the work with some perspectives.

\section{Our Case Study-Limitations}

When a person is moving in an unknown area and making a search, only maps can provide desired information in a precise and concise way. Google Maps, Bing, Via Michelin, Mappy, OpenStreet-Map, and many others are cartographic publishers trying to visualize the geographic information on 2D/3D base-maps. However, most of them are still retrieving data from one single GDB. What if this GDB is not updating its information very often (Google street view case) or had been hacked and many details were changed? Collecting information from many GDBs is trustworthy and more accurate if we know already how to deal with some inconsistencies between homologous objects, candidates for integration.

The scope of our work is to ensure location and cartographic integrations for LBS information supplied by many providers. In other words, homologous objects should be integrated to avoid duplicated icons on the mobile screen. Some deficiencies might be encountered as detailed in (Karam et al. 2010) which are related to 1) the lack of real time updates in GDBs for place names and semantic details, 2) GPS precision and different reference systems for geographic positions and 3) proprietary base maps and different legends for each service provider.

\section{Related Works}

Currently, we can find visual portals listing services from many providers in alphabetical order or according to user's preferences (Laurini et al. 2008). This interface is not user-friendly if we have a long list of services to scroll down. Another visualization is to represent services by shapes and the correspondent providers by their colors but we are limited to maximum ten colors for better perception thus ten providers. Another approach is based on a 3D perspective street view map where the services are listed as place names with arrows. Overlap of place names in $3 \mathrm{D}$ and cognitive difficulties had lead to the usage of icons instead of place names with info window to show their semantic details.

On the other hand, ontology engineering is considered as providing solutions to semantically integrate several data sources. From Artificial Intelligence to Semantic Web, conventional ontologies are defined as a collection of concepts C, In-

stances I, Properties P, Axioms A and relations defined on these concepts to represent the knowledge in a certain domain of interest and provide reasoning and 
inference mechanisms (James et al. 2010). Medellin, Serna, Vargas and Ruiz in (Medellin et al. 2009) had mentioned how the use of ontologies offers some advantages for the integration of geographic information on the web. Fonseca, Camara and Monteiro in (Fonseca et al. 2006) had considered that if the use of ontologies is part of geographic information system (ODGIS, Ontology-Driven GIS), it can present multiple interpretations or roles of a same geographic feature (e.g. lake for the department of water is different in meaning as for the environment scientist or a tourism department). ODGIS acts as a system integrator of the model with many levels (top level ontology/domain, task ontologies then application ontology). We can use ODGIS framework to interpret images or icons with other kind of geographic information in a smooth and flexible way.

In order to facilitate the user creation and edition of ontologies, tools like Protégé ${ }^{1}$ or G-Match had appeared for building and/or merging ontologies towards a domain reference one.

Many researchers had discussed the state of the art of conventional ontologies and geographic ones including tourism and made comparisons about the available tools for building and/or matching and aligning ontologies. Besides, Spatial Ontology Community of Practice $\left(\mathrm{SOCoP}^{2}\right)$ provides a good forum for exposing and coordinating geospatial ontologies.

However, due to the limitation in paper size, the related works for geo-ontologies will not be detailed in this article.

We will just focus on the novelty in this domain that could be used for our purpose.

Furthermore, we want to present what OGC had implemented as standards for generating maps:

- $\quad$ According to OGC specification, a Web Feature Service (WFS) provides an interface allowing requests for geographic features across the web using platform-independent calls. The response for a "Get Capabilities" request returns capabilities such as: name, title, longitude/latitude, etc. WFS rely on Geographic Markup Language (GML), in order to insure interoperability, but does not allow alone for semantic interoperability, thus the need for semantic integration ontology behind.

- $\quad$ The Web Map Service (WMS) is a standard protocol for serving geo referenced map images over the Internet that are generated by a map server using data

\footnotetext{
${ }^{1}$ http://protege.stanford.edu/

${ }^{2}$ http://www.socop.org/
} 
from a GIS database. It produces maps as image or SVG (Scalable Vector Graphic). Individual maps can be requested from different servers and accurately overlaid to produce a composite map via WIS (Web Integrator Service) but this type of integration will keep the copyright and other built in proprietary visual aspects of each provider's map (e.g. legend, source, scale, etc.).

- $\quad$ Another OGC standard XML-based known as Symbology Encoding (SE) gives several cartographers the ability to share the cartographic description of a map layer. An expert will predefine symbologies to be shared on a map layer and pushed them as features in WFS. With OGC tools, the cartographer can export the result of his work as XML based files which have the capacity to construct several types of maps. As example of symbolizer elements which specifies how to draw the symbol, we can cite: area, point, line, polygon, text, raster. For encoding complex line styles, colored fills, text labels, etc. (Tenet 2008) so we can easily generate adequate map-legends according to symbology encoding descriptions (feature type styles and coverage styles). What is just needed for OGC is to work on a better alignment with ISO 19117 at this level

\section{Discussion and Implemented Solutions}

After this overview, we can discuss and evaluate better our main contribution in this research area.

First of all, we decided to visualize location based services on desktop and mobile screens by contacting many service providers. In our platform, we supposed that the geographic objects are points (0D).

To ensure that two objects for the same location based service, listed by two different providers, are candidates for integration and should be visualized once, different solutions at different levels were selected and adopted:

\subsection{Section 1: Main Contribution for Location Integration}

4.1.1 At the geographic integration level, to decide whether two punctual objects are the same and need to be integrated, the Euclidian distance dE is used. So as far as the distance between object 1 and object 2 is less than a threshold of $5 \mathrm{~m}$ for example, we can then suggest that these objects are homologous. However, the choice of the threshold is very important. More details were mentioned in (Karam et al. 2010). 
4.1.2 For the place names' integration, the fusion technique uses the Levenshtein distance to compare the place names (String of characters) of two objects from two different providers (Karam et al. 2010).

4.1.3 Semantic integration between these two objects is related to their metadata/data differences (e.g. phone, email, website, etc.). To avoid duplication of the service details from two different providers, Levenshtein distance algorithm had been used to check for homologous information and the agreed results were deducted from a matching table in our framework MPLoM (Multi Providers LBS on Mobile devices). A semantic ontology-driven approach could be implemented via Protégé as a second more intelligent solution using inference reasoning. For example, if a pedestrian wants to know what restaurants can offer "Hamburger", the platform should list all the restaurants of type American or Fast food.

4.1.4 We can assume that difficulties in location integration had been partially solved by the above solutions. The final decision for homologous objects depends on the output result of the belief function with Dempster operator. Geographic positions, place names and semantic details results are assigned each one a certain weight, reflecting the degree of candidates' homogeneity towards integration. Dempster operator will combine the three different weights and as far as their sum is high, the probability to consider both (Karam et al. 2010).

\subsection{Section 2: Main Contribution for Cartographic Integration}

Once the integration problems were solved at the information level, we will consider the integration at the cartographic visual level. One should be able to visualize on the screen a unique base map whose components are retrieved from the various providers contrary of what is shown in Fig. 2 and 3 below. 


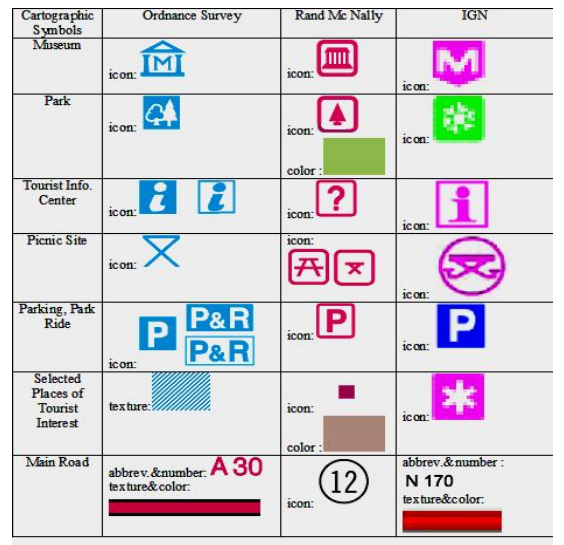

Fig. 2 Excerpts of legends from three different providers: Ordnance Survey, Rand McNally and IGN

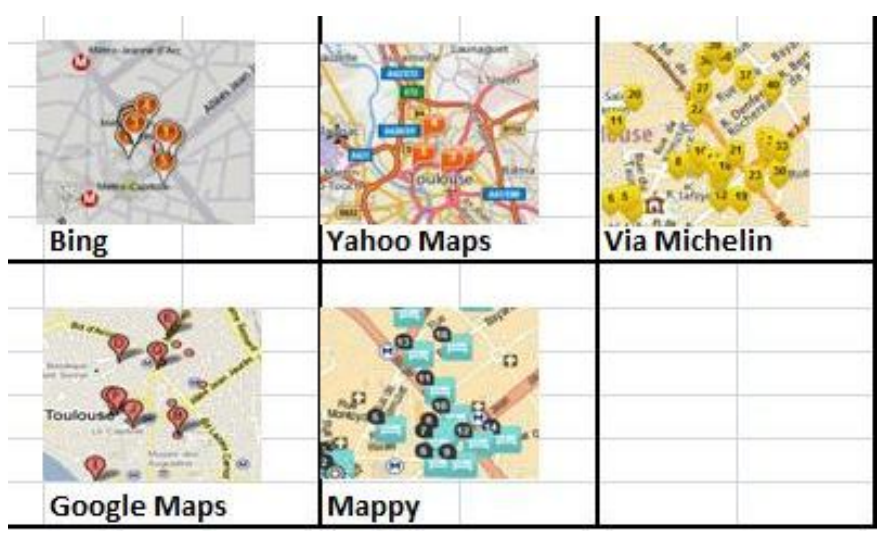

Fig. 3 Hotels in Toulouse from different portrayal layouts

Two different scenarios could be implemented: Symbology Encoding or CartOntology with visual concepts. We had taken the challenge to propose and implement the second scenario.

\subsubsection{Scenario 1: Applying Symbology Encoding Concept}

From (Tenet 2008) and (Ertz et al. 2010) we can notice that we can build up the map context as below:

- $\quad$ Define one or more possible base layers 
- Define one overlay for the track and one for way points

- Define a layer of point of interests (POI)

- Define the symbology of each layer by use of Symbology Encoding according eventually a set of predefined symbologies for points, lines, texts, areas, etc.

- Define initial map extent and spatial reference system

- Describe metainformation, layer name, date, author, source, orientation, legend, etc.

The XML SE file which is the dictionary collection of the POI visual attributes (icon, color, texture, number, label, etc) can be inserted as part of WFS file that includes via GML tags all the semantic details and geographic coordinates of the different POIs.

WMS will then visualize on the collected base maps, the mash ups of the POIs retrieved from WFS file with SE visual symbols. The author name, source, date, orientation, scale, legend, etc are inserted as modules on the base-map.

The drawback of this solution is that each base-map will include its own metadata and if we want to contact many providers, the integration of many base-maps via WMS will keep the copyrights for each map provider such as source, legend, date, etc. for marketing purpose. Besides, the symbology encoding XML file is defined manually by the admin-cartographer as reference and we can't accept the proprietary symbols of each provider. Otherwise, we must include a knowledge database to collect and match all the Symbology Encoding files from the different providers towards a domain global one. This knowledge database is a kind of geoontology for icon-based retrieval that we decided to implement it in our solution.

\subsubsection{Scenario 2: Applying a new type of Ontology with visual concepts}

From (Domingues et al. 2009), we can find that it is possible to develop a geoontology framework for color assignment to maps on demand. In order to develop automatically a map based on the graphical semiology rules, the users' preferences for colors and the outputs of the Chromatic Circle (Chesneau, 2006) and Derain Algorithm (Derain, 1905), Domingues et al. had implemented ontology of colors for this purpose.

(James et al. 2010) had proposed directions for the application of ontology matching techniques to solve different interoperability issues in the area of image annotation and retrieval so we can replace 'image' with 'icon or texture' for example and test the feasibility of their framework. In the context of semantic image annotation, ImageNet and LSCOM are two examples of multimedia ontologies where the concepts are the nodes of the WordNet ontology and the instances are 
the images, or the visual attributes in our case, in the associated databases labeled by these concepts.

We can deduce that ontologies are convenient to represent visual knowledge or map legend but we should bridge the semantic gap problem between the semantic level and the visual level representations. This can be solved by 1) matching ontologies at the semantic level with ontologies at the visual level, and 2) matching multiple visual ontologies in order to extract a common visual model for linguistic descriptions of images or icons.

Besides, we can apply "variable selection techniques" in machine learning that can serve to rank the input variables (for example, the different icons for same POI service) by their importance for the output visualization, according to user's evaluation criteria, his context/profile and other semiology constraints. Belief weights could be applied within OWL file (BeliefOWL) for each symbol to ensure ranking as well.

We decided then to develop a new type of geographic ontology framework to build and match semantic and visual aspects of the providers' legends towards a domain reference one for LBS. Because we are dealing with ontologies, we will use the tags 'properties' of OWL (Web Ontology Language) standard to include the visual attributes of each POI concept such as his icon, color, texture, font, number, etc. instead of normal XML tags as per SE. Other interpretation was to extend OWL with a new tag called Symbol in order to code the visual aspects for each concept as detailed below. This later is implemented in the platform.

By respecting the proposed paradigm: "the visual ontology of concepts", each provider shall have its own local ontology that should be populated via a Graphical user interface (GUI) implemented for this purpose. This is done by inserting manually their semantic and visual service attributes based on their legends, semantic concepts for the name of POIs and aside the visual aspects of the correspondent symbol.

As OWL can handle only textual concepts, it needs to be revised in order to describe visual aspects as well. (Karam et al. 2010) had well explained our scenario. A more invasive suggestion is to propose an extension of OWL standard; we named it CartOWL in order to describe in a dedicated and organized file all the visual concepts and their relationships of equivalence and inclusion.

Once the local ontologies corresponding to the LBS providers' cartographic visual concepts are generated, then the matching/alignment step should start. 
The full prototype will be able to parse the CartOWL output files and align them towards one reference knowledge base (domain ontology) so that we can ensure map conflation results on mobile devices.

Belief function must be applied as well through CartOWL in order to achieve the best compromise between the domain ontology and other constraints that may interfere such as the user's profile (nationality, map preference, age, etc.), the context of his geographic zone, the graphical semiology rules and color contrasts v/s visibility, the device limitations and the need for generalization, adaptation and dynamic maps, etc. So, in order to prioritize visual attributes from one provider among others, highest weights will be assigned to them as per the belief theory in the CartOWL tags. Psycho-cognitive test for efficient icons-recognition, without legends, will help us to assign such degrees of preference or weight and prioritize an icon among many representing the same service.

Some screenshots of our building/matching prototype are shown below:

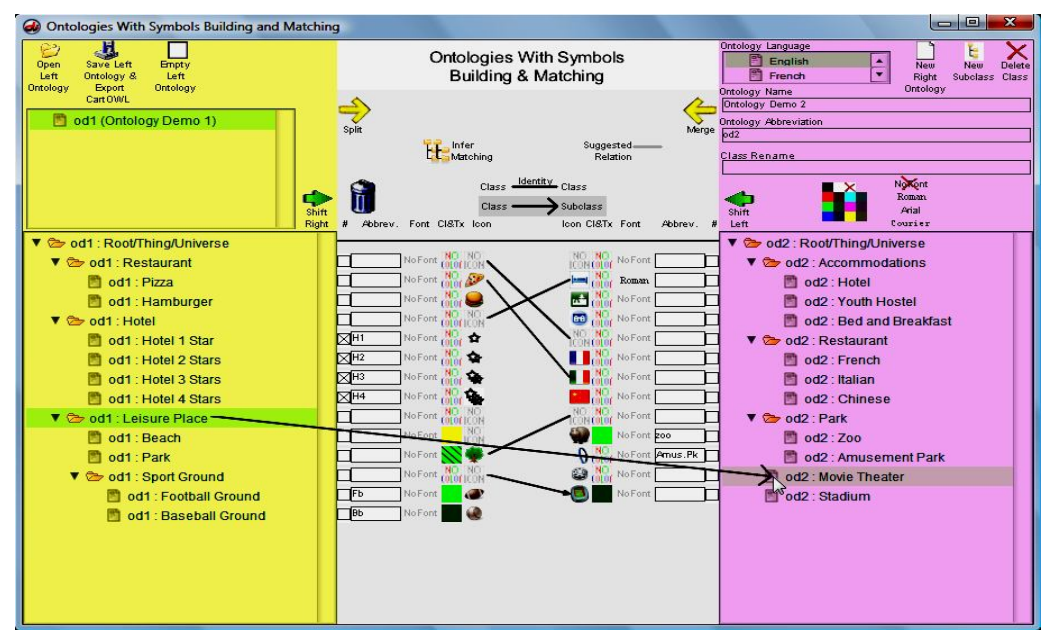




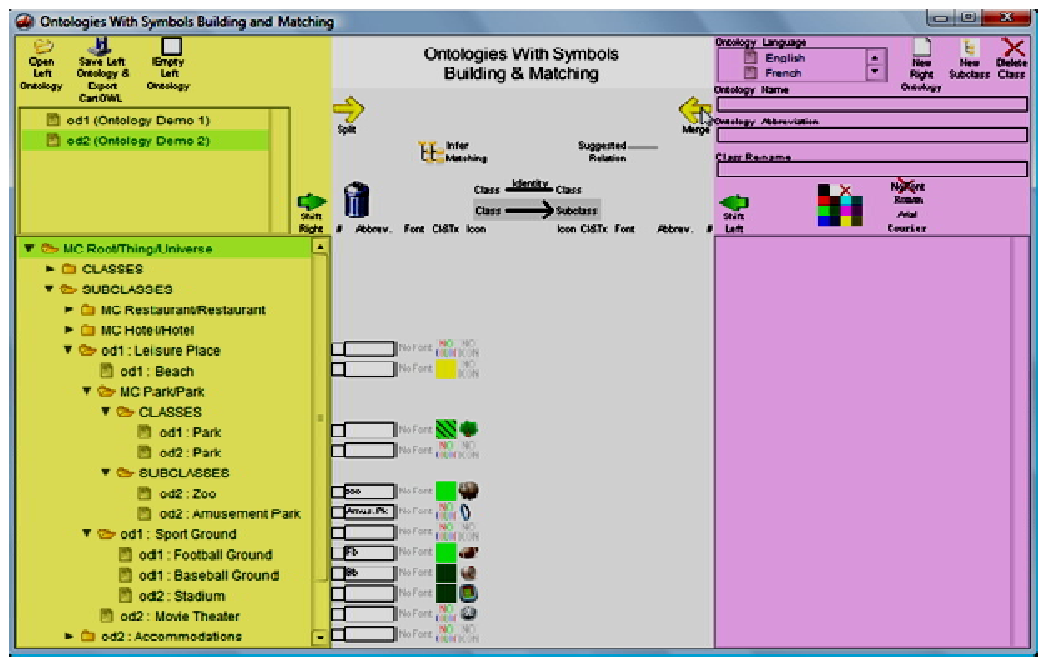

Fig. 4 Building/Matching part of the application (focus on the "Leisure Place" class)

Finally, we had adopted this scenario comparing to the SE (Muller 2006) and OGC standards for the following reasons:

1. We don't neglect the proprietary visual aspects and legends for each provider and his base map so that the provider, the administrator and the user can benefit from this work.

2. We don't ask the provider to build his own XML file dictionary as for the SE standard. However, the administrator had been charged to do this manually by referring to his map legend only.

3. For scalability reasons, it is more efficient to go for a semi to automatic framework matcher. The administrator will then ask each provider to give him its OWL file of legend symbols easily built from a visualization interface such as Protégé.

4. Our Framework have the import/export tool for OWL files and can easily match the semantics at the concepts level then the visual aspects at instances or properties levels between two ontologies.

5. Our framework will align any new ontology with the resulted global one automatically. 
The final OWL file named CartOWL will include all the referenced symbols as XML based (same idea as Symbology Encoding XML file). However, this file will be adjusted as well based on users' profile ontology (age, nationality country, culture) for the choice of adequate icons/base map and the graphical semiology rules applied in the color ontology developed by (Domingues et al. 2009) for the choice of colors.

For the time being, building/matching these ontologies with visual aspects is done manually by a domain expert. However, we can develop automatic reasoner and extend Protégé or G-Match in order to include the visual attributes and do the matching semantically based on concepts-names matching algorithms (word by word, keyword, external thesaurus as $\mathrm{WordNet}^{3}$, string distance matching, semantic Google distance etc) then collect the icons such as ImageNet ${ }^{4}$ labeled by the same concept name and set a certain variable weight for each icon to prioritize our selection. This weight is corresponding to the result of a psycho-cognitive test distributed to users for best icon-recognition without legends.

The complete solution as proposed above should be validated by our MPLoM platform. Its main purpose is to test the feasibility of the location and map symbols' integrations into a unique visual portal on mobile devices and desktop.

The phase 1 of MPLoM implementation covers the location integration from two different providers offering pull and push services.

The pull services which are the nearest hotels and restaurants are visualized on a 2D background Google map and the components are overlaid as Google markers( $\mathrm{R}$ for restaurants and $\mathrm{H}$ for hotels); the details for each clickable restaurant marker or hotel are presented textually on the mobile device. A user interface is created to get all the preferences of the clients (e.g. name, age, nationality, major, email, credit card, language, etc.) and save them into a middleware admin database. Thus, the client's request with all the needed parameters will be forwarded via Java servlet to the concerned tables in the providers' databases (Karam et al. 2010).

Both providers' databases are created in PostgreSQL with the PostGIS feature for spatial usage. Each user's request will be then subdivided into two sub requests, one for each provider in order to collect the available data.

Output data for each requested service type, will be saved as a GML file, precisely in cGML format (compact for mobile device). All cGML output files, one per provider, will be collected in the middleware admin database. XQuery, from W3C body, is used to parse these cGML files, in order to integrate the details of

\footnotetext{
${ }^{3} \mathrm{http}: / /$ wordnet.princeton.edu/

${ }^{4}$ http://www.image-net.org/
} 
homologous objects and append the heterogeneous ones into a unified cGML file response.

The MPLoM executable file, or in other words, the LBS middleware installed on the mobile device (an S60 Nokia emulator in our case), can then easily match and display on the screen, each cGML tag accordingly.

In order to implement a weather forecast push service, we choose a different approach: The MPLoM LBS middleware connects to the available weather forecast web services, which responses can be easily integrated, due to the fact that they are in $\mathrm{xml}$ format.

We had implemented as well a catalog service in the mediator database to list all the metadata about the providers and their offered services (service type, free or not, the frequency of updates, covered countries, languages, etc.). This kind of metadata catalog would be very useful: based on the user's request and preferences, we can implement a pre filter step, in order to access only the adequate providers, and thus minimizing the response delay on the screen.

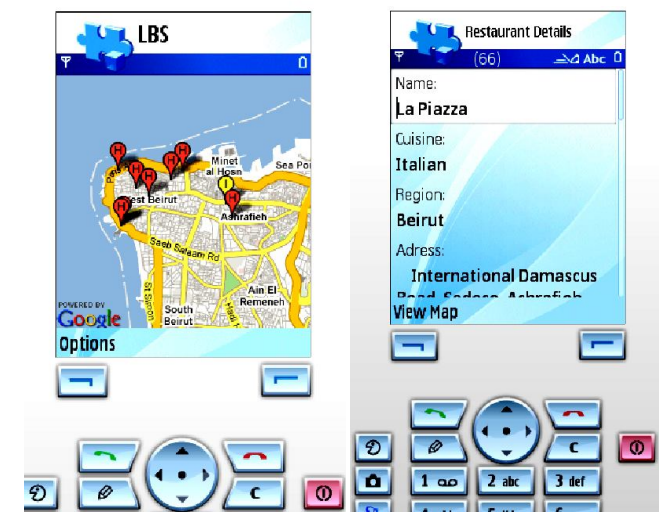

Fig. 5 MPLoM phase 1: Nearest LBS Hotels on Nokia Emulator with only Location integration

For Phase 2, we used the output of the domain visual ontology file CartOWL and we parse it with XQuery so we can visualize the correspondent symbols for each point of interest on the screen. This is the same idea between WFS and Symbology Encoding file. Unified CGML and unified CartOWL files will present the total mash ups on the adequate base map. 
However, for integrated homologous objects, we collect all their icons into one macro icon with Halo around and an aggregator sign "+" which means that their information are integrated and they are included in the info window. The user can at any time switch back to know the details from a certain provider rather than the integrated result.

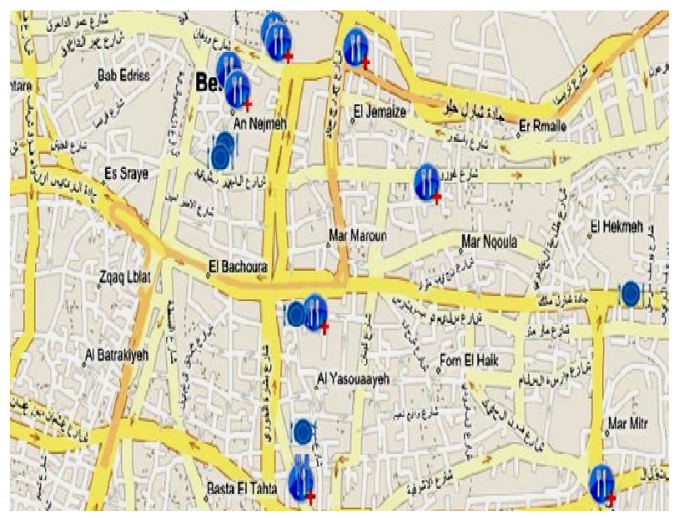

Fig.6 MPLoM phase 2: Nearest LBS Restaurants on Desktop PC with Location and Cartographic integration (Base Map for Bing 2D)

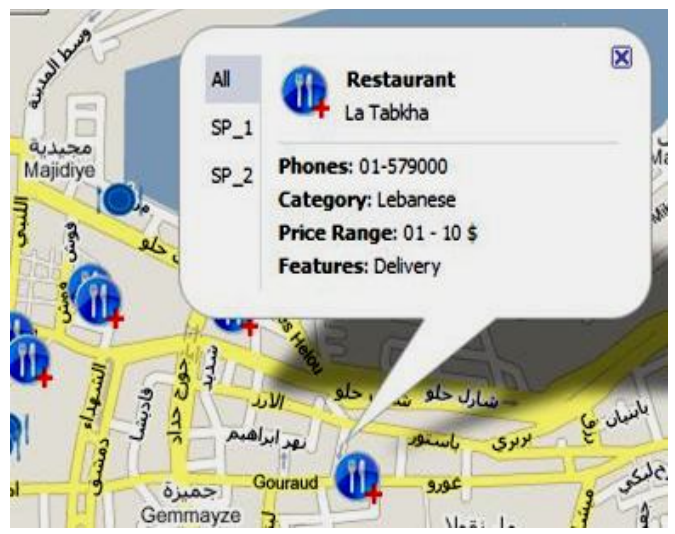

Fig. 7 MPLoM phase 2: Nearest LBS Restaurants on Desktop PC with Location and Cartographic Integration (Base Map Google 2D) and Info Window for ALL providers 


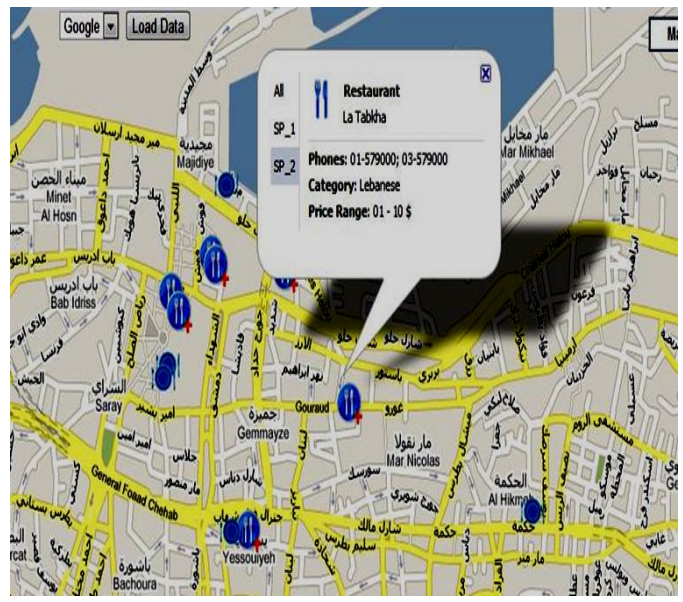

Fig. 8 MPLoM phase 2: Nearest LBS Restaurants on Desktop PC with Location and Cartographic integration (Base Map for Google 2D) Info window from SP2

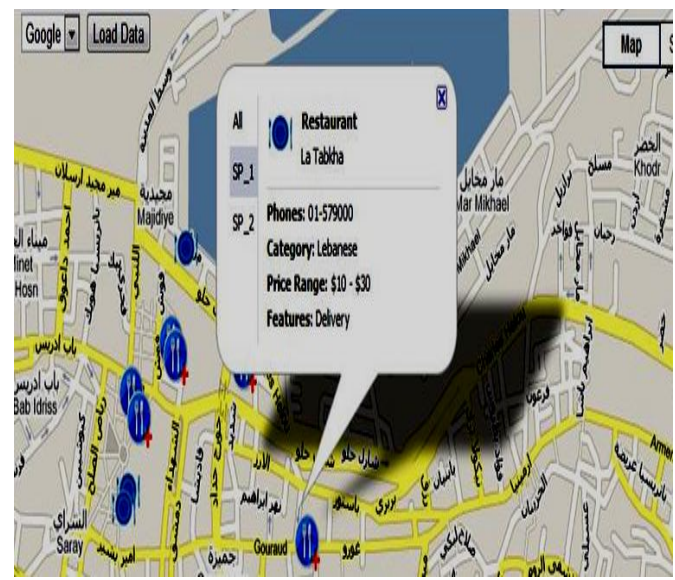

Fig. 9 MPLoM phase 2: Nearest LBS Restaurants on Desktop PC with Location and Cartographic integration (Base Map for Google 2D) Info window from SP1 


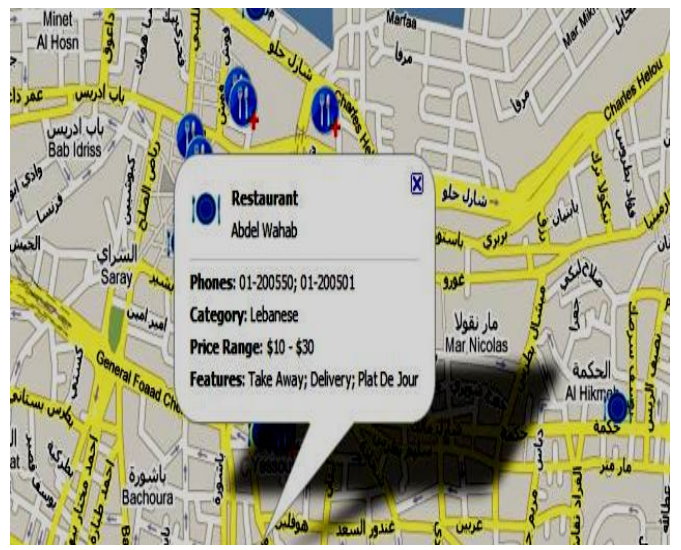

Fig. 10 MPLoM phase 2: Nearest LBS Restaurants with Info window for one different object

\section{Conclusions and Future Work}

In this paper, we presented MPLoM, a platform we had implemented, to test the feasibility of location and cartographic integrations for the same service listed by many providers on a mobile screen. Belief theory, geo ontology, geo web services and other fusion reasoning are mainly used. We also suggested many solutions to achieve the interoperability of geographic databases at the application layer. Comparing our approach to OGC standards, we believe that Symbology Encoding can play a backup solution for common dictionary of symbols instead of implementing our building/matching ontology framework with CartOWL output file. Besides, our Unified Compact GML file will contain same features but in compressed tags as WFS file. Finally, instead of calling WMS for mapping purpose, we had implemented via JavaScript and AJAX, our MPLoM source code for mash ups on Google maps and Bing.

Future enhancements should be done: 1) for the extension of G-MATCH (Zhou 2003) or Protégé open source to include visual concepts such as color, icon, texture, number, etc, and not only semantic ones and being able to do the geographic auto- matching for semantic and visual concepts without any problem, 2) for the development of Visual Attributes recognition algorithms for automatic legendbased retrieval as per J. Bertin's knowledge concerning visual variables (orientation, texture, hue, shape, etc.) and human perception (map semiotics) and 3) for the development of composite geographic web services towards a complete interoperability without any human intervention and the semantic geo web services 
domain ontology to perform matchmaking between the descriptions of a required service and the advertised ones with intelligent orchestration.

\section{References}

C. Domingues, S. Christophe, L. Jolivet : " Connaissances opérationnelles pour la conception automatique des légendes de cartes", 20èmes Journées Francophones d'Ingénierie des Connaissances (IC'09), (2009).

O. Ertz, E. Bocher et al.: "Standard-centric authoring and publication for cartographic content", in the proceedings of the 1st International Workshop WebMGS, Como, Italy, (2010).

F. Fonseca, G. Camara, A. Monteiro: "A Framework for Measuring the Interoperability of Geo-Ontologies", Spatial Cognition \& Computation, Vol. 6 (No. 4), pp. 309-331, (2006).

N. James, K. Todorov, C. Hudelot: "Ontology Matching for the Semantic Annotation of Images", IEEE International Conference on Fuzzy Systems, WCCI Conference, pp. 2499-2506 (2010).

R. Karam, F. Favetta, R. Kilany, R. Laurini: "Integration of Similar Location Based Services Proposed by Several Providers ", 2nd International Conference NDT 2010: Network and Digital Technologies, Springer Ed., pp. 136-144, Prague, (2010).

R. Karam, F. Favetta, R. Kilany, R. Laurini: "Cartographic Integration on mobile devices from several providers' LBS by means of map symbol ontology.", in the proceedings of the 1st International workshop WebMGS, Como, Italy, (2010).

R. Karam, F. Favetta, R. Kilany, R. Laurini: " Uncertain GeoInformation Representation and Reasoning: a Use Case in LBS Integration ", 5th International Workshop FlexDBIST, DEXA 2010, IEEExplore Ed., pp.313-317, Bilbao, Spain, (2010).

R. Karam, F. Favetta, R. Kilany, R. Laurini: "Integrating many Legends through ontology for cartographic symbols." in the proceedings of the 6th International Conference SAGEO2010, Toulouse, France, (2010). 
R. Laurini, S. Ordillo, C. Mostaccio, F. Raffort, S. Servigne: "Towards multiprovider LBS visual portals" ,The 14th International Conf. on Distributed Multimedia Systems, USA, pp. 208-213, (2008).

R. Medellin, G. Serna, R. Vargas, L. Ruiz: " Technology Integration around the Geographic Information: A State of the Art ", IJCSI International Journal of Computer Science Issues, Vol. 5, pp. 17-26, (2009).

M. Muller: "Symbology Encoding Implementation Specification", OpenGIS Implementation Specification, (2006).

Tenet Technology: "Some Unresolved Issues with the OGC Symbology Encoding (SE) ", submitted to OGC, (2008).

N. Zhou: "A study on automatic ontology mapping of categorical information", Proceedings of the 2003 annual national conference on Digital government research, pp.1-4, (2003). 